\section{In the news}

\section{POOR DEVILS}

The facial tumours that have decimated the Tasmanian Devil population are infectious and derive from a single animal, according to research published in Nature (http:// www.nature.com, 2 February 2006).

In the past 10 years the population of these carnivorous marsupials has decreased from 140,000 to 80,000 because of a facial tumour that causes starvation. The new research proposes that it is the animals' vicious behaviour that is to blame. "Devils jaw wrestle and bite each other a lot....and bits of tumour break off one devil and stick in the wounds of another" said the study's author, Anne-Marie Pearse (http://news.bbc. co.uk, 2 February 2006). She added "It occurred to me that there didn't necessarily have to be a virus if the [cancer] cells themselves could be transmitted" (http://www.

sciencenews.org, 2 February 2006).

The evidence that the tumours are infectious and derive from a single source came from examining karyotypes. The authors found exactly the same complex set of chromosomal rearrangements in the tumours of all 11 animals that they studied. In addition, one animal had a chromosomal rearrangement in its normal cells that was not found in its tumour. This identical tumour karyotype, found at all stages of tumour development and in all individuals, strongly supports the infectious theory.

It is thought that the low genetic diversity of the devils is the reason that the immune system does not kill the foreign cancer cells. A similar phenomenon has been observed when cancer cells are inadvertently transmitted with donated organs between closely related individuals.

A cull of infected animals is now thought to be the best way to proceed. "This is an incredibly urgent problem" said lan Campbell, the Australian Environment Minister, announcing increased funding (http://www.abc.net.au.com, 7 February 2006)

Patrick Goymer

SIGNALLING PATHWAYS

A flying start

Activation of conserved developmental signalling pathways, such as Notch, has been observed in human cancers, but activation of such pathways in animal models seems to be insufficient for tumorigenesis. Ferres-Marco et al. now report that in a Drosophila melanogaster model the Notch pathway collaborates with epigenetic-silencing pathways and cell-cycle control in tumour development.

The developing eye in Drosophila is a good model for identifying tumour-inducing mechanisms because it is simple and genetically well-defined. The growth of the eye depends on Notch activation by its ligands Delta and Serrate. The 'large eye' phenotype model, produced by overexpression of Delta (see figure), was screened by upregulating genes at random and looking for induced tumour growth. Ferres-Marco and colleagues found that overexpression of two neighbouring genes, longitudinals lacking (lola) and pipsqueak ( $p s q)$ caused tumour formation (see figure). Both Psq and Lola behave as epigenetic silencers of the Polycomb group, which maintain transcriptional repression patterns.

So, do psq and lola contribute to the tumour phenotype when coactivated with the Notch pathway? The authors introduced point mutations into these genes to disrupt their expression - all $p s q^{-}$mutations prevented tumorigenesis and all lola- mutations reduced eye-tumour size. Most of the mutations were in the BTB domain of Psq, and, as BTBprotein-family members are transcriptional repressors and include oncogenes that recruit Polycomb proteins, the authors speculated that deregulated $p s q$ and lola could lead to tumorigenesis by epigenetic

\title{
TUMORIGENESIS
}

\section{Culpable kinase}

Cyclin-dependent kinase 4 (CDK4) has been implicated as a potential new therapeutic target in women with breast cancers that overexpress ERBB2 and cyclin D1.

CDK4 regulates cell-cycle progression by interacting with the D-type cyclins and phosphorylating and inhibiting the retinoblastoma protein (RB). Cyclin D1 is overexpressed in many cancers, including most breast cancers, and loss of this protein is known to suppress mammary carcinogenesis in mouse models. However, whether this is dependent on the kinase activity of the cyclin
D1-CDK4 complex or on cyclinD1 kinase-independent functions was unclear, so $\mathrm{Yu}$ and colleagues investigated.

To check whether CDK4 activity was required for breast development, the authors used a mouse model in which cleared mammary fat pads were transplanted with either Cdk4-null mammary epithelium or wild-type mammary epithelium. All of the transplanted mice developed normal breast architecture during pregnancy and were able to lactate, supporting the
CDK4 might prove to be a selective therapeutic target in all tumours that overexpress ERBB2

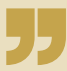

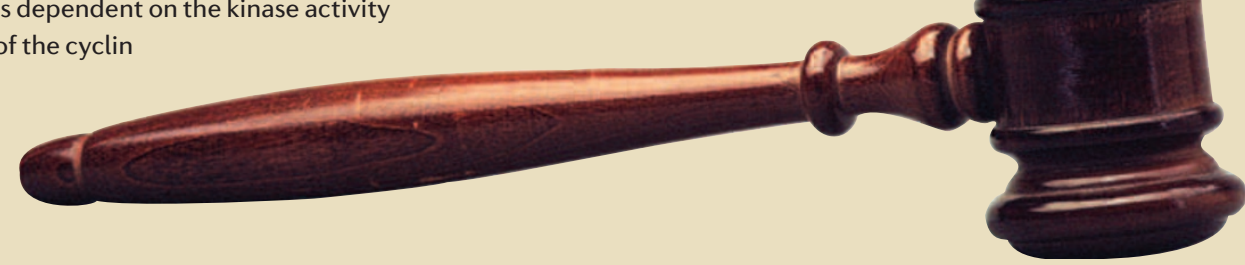


silencing. As methylation of histone $\mathrm{H} 3$ is a central modification in epigenetic control, the authors immunolabelled eye discs with antibodies against histone $\mathrm{H} 3$ methylation. A loss or reduction of $\mathrm{H} 3$ lysine-4 methylation was observed in the tumour eye discs, and overexpression of Delta significantly reduced H3 methylation too.

The next step was to look at which genes were aberrantly silenced. Such genes are likely to include those involved in the control of the cell cycle, so the authors looked at the transcription of 12 tumour-related genes in the mutant and wild-type eye discs. Transcription of $R b f$, a fly homologue of the human retinoblastoma tumour-suppressor gene $(R B)$, was strongly down-regulated in the tumours. Halving Rbf gene dosage enhanced tumour growth, and reestablishing $R b f$ expression in the eye prevented tumour formation.

These results establish a mechanism that links the Notch-Delta pathway, epigenetic-silencing pathways and cell-cycle control in the process of tumorigenesis.

Ezzie Hutchinson

\section{ORIGINAL RESEARCH PAPER Ferres-Marco, D.} et al. Epigenetic silencers and Notch collaborate to promote malignant tumours by $\mathrm{Rb}$ silencing. Nature 439, 430-436 (2006)

\section{Control eye}

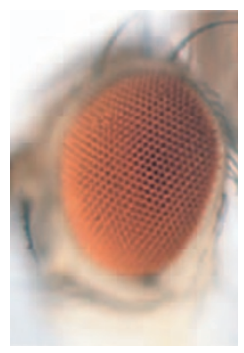

Delta overexpression, showing 'large eye'

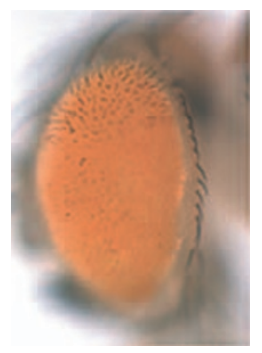

Delta, psq and lola overexpression, showing tumour growth

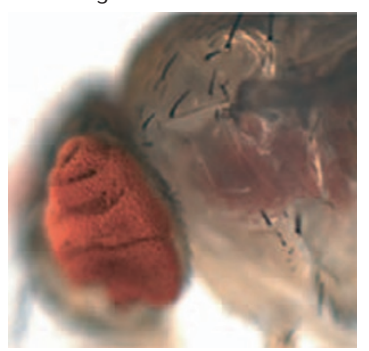

previous finding that expression of cyclin D1 is not required for breast development.

To investigate the function of CDK4 in breast tumorigenesis, the authors crossed $C d k 4$-null mice with mice expressing the oncogene Erbb2 under the control of the mouse mammary tumour virus promoter. They found that loss of CDK4 inhibited breast tumour development.

As well as inactivating RB, cyclin D1-CDK4 complexes sequester p27 from cyclin E-CDK2 complexes to enable cell-cycle progression. To investigate whether this function was important for tumorigenesis, the authors collaborated with Landis and colleagues to produce cyclin-D1 knock-in mice that express a cyclin-D1 mutant protein that is still able to interact with CDK4 and so sequester p27, but the complex has no kinase activity. These mice were also resistant to Erbb2-driven mammary tumorigenesis, indicating that it is the kinase activity of this complex that is required to induce $E r b b 2$ breast tumours. Yu and colleagues also used RNA interference to show that CDK4 kinase activity is required to maintain the tumours as well as initiate them.

Does this model hold true in human breast cancer? By analysing protein expression levels in 70 sections of human breast tumours that overexpress ERBB2, the authors found that $27 \%$ showed increased expression of cyclin D1. Because most tissues develop normally in both Cdk4-null and cyclin D1-null mice, the authors suggest that CDK4 might prove to be a selective therapeutic target in all tumours that overexpress ERBB2.

Nicola McCarthy

ORIGINAL RESEARCH PAPER Yu, Q. et al. Requirement for CDK4 kinase function in breast cancer. Cancer Cell 9, 23-32 (2006)| Landis, M. W., Pawlyk, B. S., Li, T., Sicinski, P. Hinds, P. W. Cyclin D1-dependent kinase activity in murine development and mammary tumorigenesis. Cancer Cell 9, 13-22 (2006)

\section{IN BRIEF}

\section{TARGETED THERAPY}

mTOR inhibiton induces upstream receptor tyrosine kinase signaling and activates Akt.

O'Reilly, K. E. et al. Cancer Res. 66, 1500-1508 (2006)

Stimulation of insulin-like growth factor 1 (IGF1) receptor induces the phosphatidylinositol 3-kinase (PI3K)-AKT-mTOR pathway, which is frequently activated in cancer cells. mTOR inhibition can arrest tumours in model systems, but shows limited anti-tumour activity in patients. O'Reilly et al. show that mTOR inhibition induces the expression of insulin-receptor substrate 1, which, in turn, induces IGF1 receptor activity. This upregulates AKT and negates the effect of mTOR inhibition. This feedback mechanism might explain the poor therapeutic effect of mTOR inhibitors. Furthermore, IGF1-receptor inhibition is shown to sensitize cells to mTOR inhibition, indicating promise for future combination therapies.

\section{$\Rightarrow$ METASTASIS}

Role of $\beta$-arrestin 1 in the metastatic progression of colorectal cancer.

Buchanan, F. G. et al. Proc. Natl Acad. Sci. USA 103, 1492-1497 (2006)

The transactivation of growth-factor receptors by ligandactivated G-protein-coupled receptors (GPCRs) has been implicated in human cancer-cell survival, proliferation and migration. Now, Raymond DuBois and colleagues show that $\beta$-arrestin is involved in the SRC-dependent pathway through which this transactivation occurs. The association of a ligandactivated GPCR- $\beta$-arrestin-1-SRC signalling complex results in transactivation of the epidermal-growth-factor receptor (EGFR) and downstream AKT signalling. Furthermore, the $\beta$-arrestin-1-SRC interaction is required for both colorectal carcinoma cell migration in vitro and metastatic spread of the disease in vivo.

\section{$\Rightarrow$ IMMUNOLOGY}

Autoimmunity and tumour immunity induced by immune responses to mutations in self.

Engelhorn, M. E. et al. Nature Med. 29 Jan 2006 (doi:10.1038/nm1363) Weakly immunogenic tumours can be converted to strongly immunogenic ones by exposure to mutagens, and mutagenized tumours can induce immunity against the original tumour in mouse models. The basis for this is unclear, but work from Alan Houghton and colleagues indicates that specific mutations can alter the intracellular trafficking routes of proteins. This means that the mutant proteins are more likely to be presented as antigens rather than recognized as self peptides.

\section{$\Rightarrow$ CHROMOSOMAL TRANSLOCATIONS}

\section{H2AX prevents DNA breaks from progressing to} chromosome breaks and translocations.

Franco, S. et al. Mol. Cell 21, 210-214 (2006)

This paper shows that histone $\mathrm{H} 2 \mathrm{AX}$, a protein involved in the repair of DNA double-strand breaks, is an essential component for inhibiting chromosomal translocations that involve the immunoglobulin-heavy-chain locus $(\mathrm{lg} H)$ in activated B cells. Importantly, the authors conclude that mutation of the tumour suppressor and DNA-damage-response protein p53 does not influence the frequency of such $\mathrm{lg} H$ translocations, but instead might enable the expansion of $B$ cells that harbour them. 